\title{
Direct evidence of the low-temperature cluster-glass magnetic state of $\mathrm{Nd}_{2 / 3} \mathrm{Ca}_{1 / 3} \mathrm{MnO}_{3}$ perovskite
}

\author{
Alexander Feher ${ }^{1}$, Vladimir Desnenko ${ }^{2}$, Elena Fertman ${ }^{2}$, Sergiy Dolya $^{2}$, \\ Marcela Kajňaková ${ }^{1}$, and Anatoly Beznosov $^{2}$ \\ ${ }^{1}$ P.J. Šafárik University in Košice, Faculty of Science, \\ Park Angelinum 9, 04154 Košice, Slovakia \\ ${ }^{2}$ B. Verkin Institute for Low Temperature Physics and Engineering of the National Academy of Sciences of Ukraine \\ 47 Lenin Ave., Kharkov 61103, Ukraine \\ E-mail: fertman@ilt.kharkov.ua
}

Received February 13, 2012

\begin{abstract}
In the presented study we have revealed a giant exchange bias in a colossal magnetoresistance $\mathrm{Nd}_{2 / 3} \mathrm{Ca}_{1 / 3} \mathrm{MnO}_{3}$ perovskite at low temperatures, evident of an intrinsic exchange coupling in this compound. The phenomena found confirms our previous assumption that the low-temperature magnetic structure of the compound is represented by small (nanosized) ferromagnetic clusters immersed within the charge-ordered antiferromagnetic matrix. Magnetic behavior of the $\mathrm{Nd}_{2 / 3} \mathrm{Ca}_{1 / 3} \mathrm{MnO}_{3}$ perovskite is consistent with a cluster-glass magnetic state and does not agree with a classical spin-glass state observed in a variety of disordered magnetic systems. We think that the cluster-glass magnetic behavior of $\mathrm{Nd}_{2 / 3} \mathrm{Ca}_{1 / 3} \mathrm{MnO}_{3}$ originates from the selforganized phase-separated state of the compound. The Cole-Cole analysis of the dynamic susceptibility at lowtemperatures has shown extremely broad distribution of relaxation times, indicating that spins are frozen at a "macroscopic" time scale. Slow relaxation of the zero-field-cooled magnetization has been experimentally revealed as well. This slow relaxation confirms the cluster-glass magnetic state of the compound. Two strongly different relaxation mechanisms were found: the first one is characteristic for temperatures below the freezing temperature $T_{g} \sim 60 \mathrm{~K}$, the second one is characteristic for higher temperatures.
\end{abstract}

PACS: 64.75.-g Phase equilibria;

75.50.Lk Spin glasses and other random magnets;

75.30.-m Intrinsic properties of magnetically ordered materials;

75.47.Lx Magnetic oxides;

77.22.Gm Dielectric loss and relaxation.

Keywords: cluster glass, exchange bias, phase separation, relaxation.

\section{Introduction}

Insulating $\mathrm{Nd}_{2 / 3} \mathrm{Ca}_{1 / 3} \mathrm{MnO}_{3}$ perovskite is a colossal magnetoresistance narrow-band perovskite compound which shows phase-segregated state at low temperatures. The charge ordering phase transformation in $\mathrm{Nd}_{2 / 3} \mathrm{Ca}_{1 / 3} \mathrm{MnO}_{3}$, which takes place at $T_{c o} \approx 212 \mathrm{~K}$ [1], is of the first-order martensitic type. It leads to the self-organized coexistence of charge-ordered (CO) and charge-disordered (CD) phases in a wide temperature range below the room temperature. Extended temperature hysteresis of the magnetic susceptibility in the charge ordering region is one of the evidences of the nonequilibrium phase-segregated state [1].
Below $T_{c o}$ the compound exhibits a sequence of magnetic transformations: two antiferromagnetic ones at $T_{N 1} \sim 130 \mathrm{~K}$ and $T_{N 2} \sim 80 \mathrm{~K}$, and a ferromagnetic one at $T_{C} \sim 70 \mathrm{~K}[2,3]$. They lead to the coexistence of at least three different magnetic phases at low temperatures: two antiferromagnetic charge-ordered ones (AFM) (their fraction is about $82 \%$ ) and the ferromagnetic (FM) chargedisordered one (its phase fraction is about 18\%) [4]. Furthermore, the low-temperature magnetic phase-segregated state arises from the $\mathrm{CO}-\mathrm{CD}$ phase segregated state: $\mathrm{CO}$ phase becomes antiferromagnetic, $\mathrm{CD}$ phase becomes ferromagnetic. 
In recent years, unusual nonequilibrium dynamics and time-dependent phenomena in phase-segregated perovskites have been reported [4-8]. In particular, slow relaxation and memory effects in magnetization, the frequencydependent ac susceptibility and aging were found. The glassy behavior in the phase-separated perovskites is strongly associated with the coexistence of different magnetic phases and therefore such compounds do not behave like canonical spin glasses, they rather have to be considered as self-organized assembly of interacting magnetic clusters.

In the present work we have investigated magnetic behavior of $\mathrm{Nd}_{2 / 3} \mathrm{Ca}_{1 / 3} \mathrm{MnO}_{3}$ perovskite, and we have shown an exchange bias at low temperatures, which we take as a direct evidence of an intrinsic exchange coupling in the compound and the presence of small ferromagnetic clusters immersed in the charge-ordered antiferromagnetic phase. The magnetic phase separation at low temperatures leads to a cluster-glass magnetic state of the compound.

\section{Experiment}

A ceramic $\mathrm{Nd}_{2 / 3} \mathrm{Ca}_{1 / 3} \mathrm{MnO}_{3}$ sample was prepared by the standard solid state reaction technique from stoichiometric amounts of proper powders. The x-ray crystalstructure analysis indicated a single-phase material at the room temperature.

Magnetic measurements were made using the quantum design magnetic properties measurement system (MPMS) and a noncommercial superconducting quantum interference device (SQUID) magnetometer.

Magnetic hysteresis loops were measured at $10 \mathrm{~K}$ by the SQUID magnetometer technique after cooling the sample in zero magnetic field and after cooling it in an applied field of $H_{\text {cool }}=800 \mathrm{Oe}$.

The in-phase, $\chi^{\prime}(T)$, and out-of-phase, $\chi^{\prime \prime}(T)$, components of the complex ac susceptibility $\chi(T)=\chi^{\prime}(T)-i \chi^{\prime \prime}(T)$, were measured by MPMS at different frequencies from 1.3 to $1116 \mathrm{~Hz}$ at a fixed ac field of $0.25 \mathrm{mT}$ in warming runs. The temperature was stabilized within $0.1 \mathrm{~K}$ at each measuring point.

\section{Results and discussion}

\subsection{Exchange bias}

One of the fundamental question of the physics of manganites is whether the phase segregated state (PSS) that develops in many systems can be described as a canonical spin-glass or it is a cluster-glass, represented by ferromagnetic entities in nonmagnetic or antiferromagnetic matrix. To answer the question we have studied the $\mathrm{Nd}_{2 / 3} \mathrm{Ca}_{1 / 3} \mathrm{MnO}_{3}$ perovskite which shows a self-organized phase-segregated state at low temperatures: two antiferromagnetic (AFM) phases and the ferromagnetic (FM) one coexist below $70 \mathrm{~K}$. The compound demonstrates features common for the glassy magnetic systems, such as a gap between zero-field-cold (ZFC) and field-cold (FC) magnetization and specific frequency dependence of the ac susceptibility [9].

In this work we have shown that the compound possesses a giant exchange bias which is specific for clusterglass systems only.

Magnetic hysteresis loops were measured at $10 \mathrm{~K}$ by the SQUID magnetometer technique after cooling the sample in zero field (ZFC) and after cooling in an applied field (FC) of $H_{\text {cool }}=800$ Oe (Fig. 1). Giant shifts along both magnetic field and magnetization axes have been found for FC but they are absent for the ZFC process that manifests the exchange bias phenomenon. This effect is a result of a unidirectional exchange anisotropy interaction, which drives the ferromagnetic clusters back to the original orientation when the magnetic field is removed.

The magnetic field induced shift of the FC hysteresis loop was determined as $H_{E B}=-\left(H_{1}+H_{2}\right) / 2 \sim 392 \mathrm{Oe}$, the coercive field was defined as $H_{C}=\left(H_{1}-H_{2}\right) / 2 \sim 139 \mathrm{Oe}$, where $H_{1}$ and $H_{2}$ are the fields at which the magnetization equals zero. The vertical magnetization shift was defined as $M_{E B}=\left(M_{1}+M_{2}\right) / 2 \sim 2.2 \mathrm{emu} / \mathrm{g}$, the magnetic coercivity was defined as $M_{C}=\left(M_{1}-M_{2}\right) / 2 \sim 1.0 \mathrm{emu} / \mathrm{g}$, where $M_{1}$ and $M_{2}$ are the magnetizations at $H=0$. The ratio $M_{E B} / M_{S} \sim 0.03$ found is large enough: its value is in a good agreement with the results for the related $\mathrm{Pr}_{2 / 3} \mathrm{Ca}_{1 / 3} \mathrm{MnO}_{3}$ compound [10], in which nanodroplets of $\sim 10 \AA$ in size are immersed in an AFM host.

Note that the exchange bias effect completely disappears at temperatures above $T_{C} \sim 70 \mathrm{~K}$, which is evidently connected with the disappearance of the ferromagnetic clusters.

The giant exchange bias revealed is evident of the lowtemperature phase-segregated state of $\mathrm{Nd}_{2 / 3} \mathrm{Ca}_{1 / 3} \mathrm{MnO}_{3}$ perovskite for AF and FM phases. Taking into account our

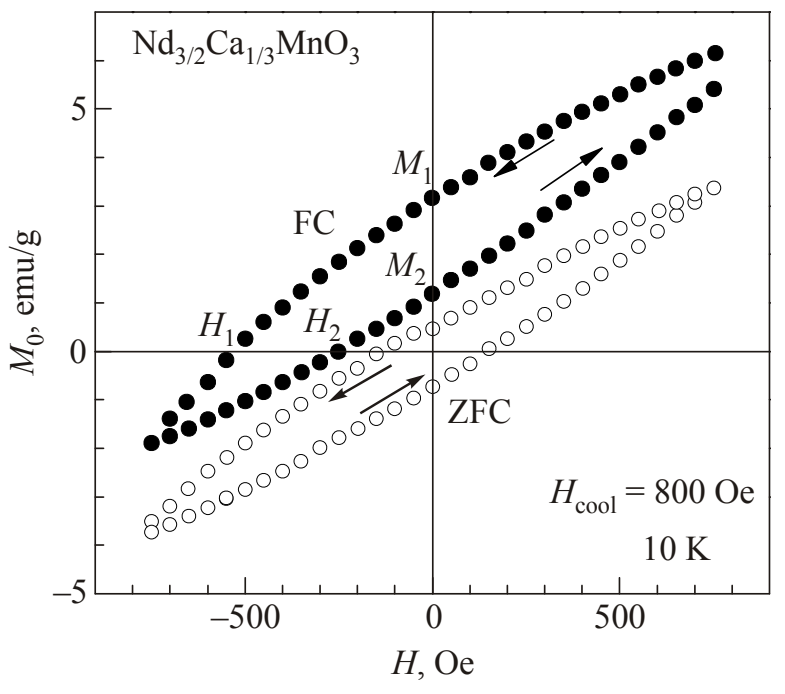

Fig. 1. Field dependence of the $\mathrm{Nd}_{2 / 3} \mathrm{Ca}_{1 / 3} \mathrm{MnO}_{3}$ magnetization at $10 \mathrm{~K}$ after ZFC and FC in $H_{\text {cool }}=800$ Oe. 
previous neutron diffraction data, showing that the ferromagnetic phase fraction is about $18 \%$ [4], we can asssume that nanosized FM clusters are immersed in the chargeordered AFM matrix.

\subsection{Relaxation of ac magnetization}

To investigate the relaxation behavior of the compound, we performed the Cole-Cole plot analysis for spin-glass systems $[11,12]$. The complex susceptibility can be phenomenologically expressed as

$$
\chi=\chi_{s}+\frac{\chi_{0}-\chi_{s}}{1+\left(i \omega \tau_{C}\right)^{1-\alpha}},
$$

where $\chi_{0}$ and $\chi_{s}$ are the isothermal $(\omega=0)$ and adiabatic $(\omega \rightarrow \infty)$ susceptibilities, respectively, $\tau_{\mathrm{C}}$ is the median relaxation time around which the distribution of relaxation times is assumed, while $\alpha(0<\alpha<1)$ is representative of the width of the distribution. From the analysis the distribution function of relaxation times at each temperature can be determined.

In Fig. 2 the temperature dependences of the real $\left(\chi^{\prime}\right)$ and imaginary $\left(\chi^{\prime \prime}\right)$ components of the ac susceptibility of $\mathrm{Nd}_{2 / 3} \mathrm{Ca}_{1 / 3} \mathrm{MnO}_{3}$ in the frequency range from 1.3 to $1116 \mathrm{~Hz}$ are shown. The $\chi^{\prime}(T)$ curves display a rather sharp peak at $T_{\max }$ which shifts upwards with increasing frequency. This is a distinct feature of both the spin-glass and the cluster-glass magnetic states [13-15]. Temperature $T_{\max } \sim 60 \mathrm{~K}$, obtained at the lowest frequency studied has been taken as the magnetic glass freezing temperature. It corresponds to $T_{g}=60 \mathrm{~K}$ obtained from our previous static magnetic measurements [9].

Temperature dependent imaginary component $\chi^{\prime \prime}(T)$ shows the corresponding shifts of $T_{\max }$. Note that the magnitude of the peak in $\chi^{\prime \prime}(T)$ increases with decreasing frequency. This is qualitatively different from the behavior of most spin-glasses in which we expect an increase of the peak magnitude with increasing frequency [16], and can be taken as an indirect evidence of the cluster-glass state of the compound studied.

The Argand diagrams for the studied compound are shown in Fig. 3, where the $\chi^{\prime}$ and $\chi^{\prime \prime}$ are taken from Fig. 2. These diagrams appear to be very unusual: there are no maxima on the diagrams at temperatures below freezing temperature $T_{g} \sim 60 \mathrm{~K}$. The "flatness" of the curves is a measure of the distribution width of relaxation times. So, the obtained data provide an evidence of a very broad distribution of relaxation times while the median relaxation time is shifted to very large values at all studied temperatures, indicating that spins are frozen at a "macroscopic" time scale. This strongly differs from canonical spinglasses $[11,12]$ and resembles a cluster-glass behavior [12].

We observe that relaxation processes below $T_{g} \sim 60 \mathrm{~K}$ and above this temperature are strongly different. At temperatures above $T_{g}$, the distribution of relaxation times

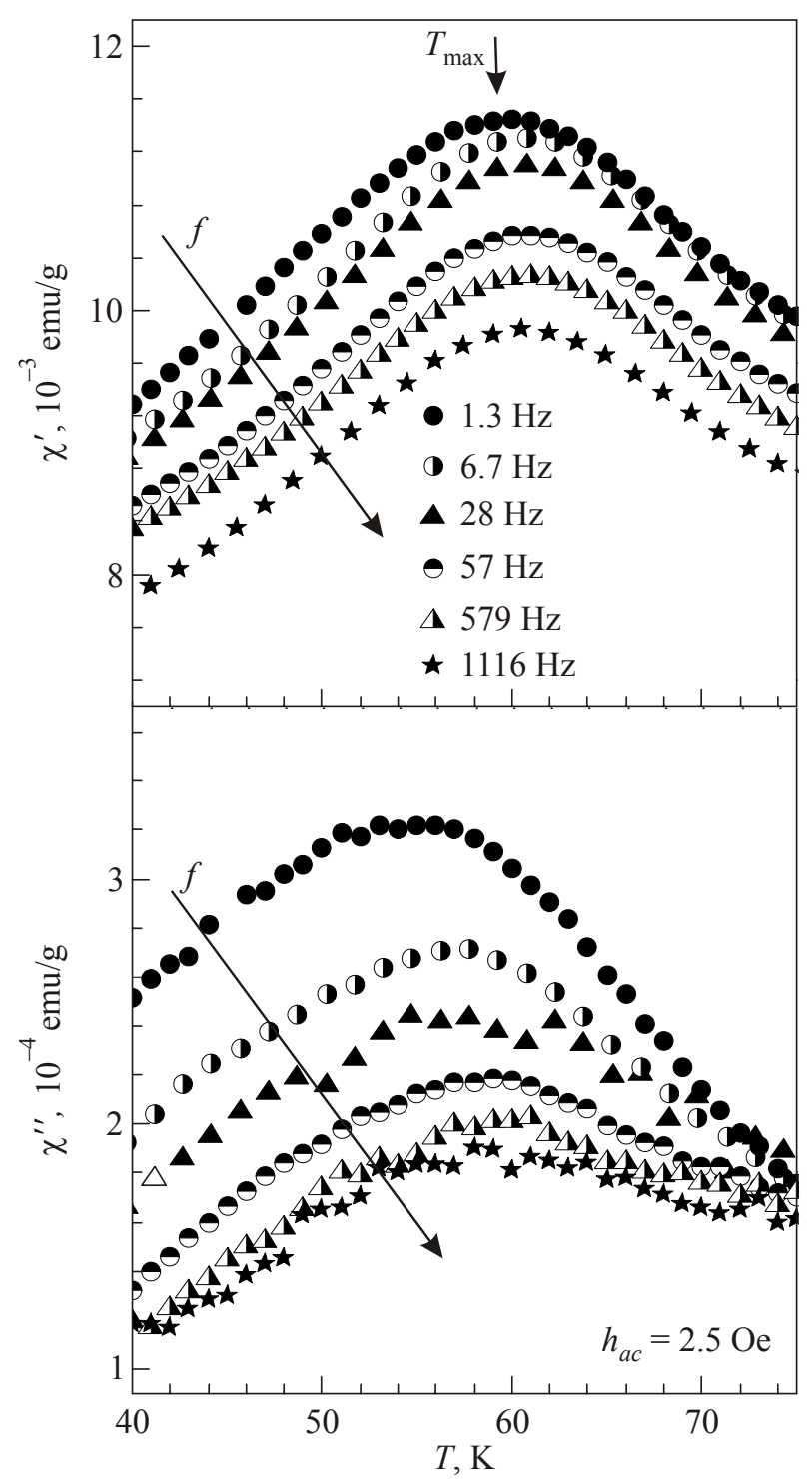

Fig. 2. Real $\left(\chi^{\prime}\right)$ and imaginary $\left(\chi^{\prime \prime}\right)$ components of the $\mathrm{Nd}_{2 / 3} \mathrm{Ca}_{1 / 3} \mathrm{MnO}_{3}$ ac susceptibility measured at different frequencies.

becomes less broad indicating the "usual" spin dynamics, while the maxima of the diagrams appear to indicate the median relaxation time (Fig. 3,b). It implies that magnetic moments of clusters are frozen at "macroscopic" time scales below $T_{g}$ and they fluctuate above this temperature.

\subsection{Relaxation of dc magnetization}

To further investigate the glassy magnetic behavior we have measured the relaxation of the ZFC magnetization. The sample was cooled from 300 to $10 \mathrm{~K}$ at $4 \mathrm{~K} / \mathrm{min}$ in zero magnetic field. Then the sample was warmed to the measuring temperature and kept at this temperature for a half an hour to allow the phase-separated state to relax. Afterwards the magnetic field $\mu_{0} H=10 \mathrm{mT}$ was applied and the magnetization data were collected immediately.

Figure 4 shows the ZFC magnetization $M(t)$ as a function of time. The magnetization data were collected 

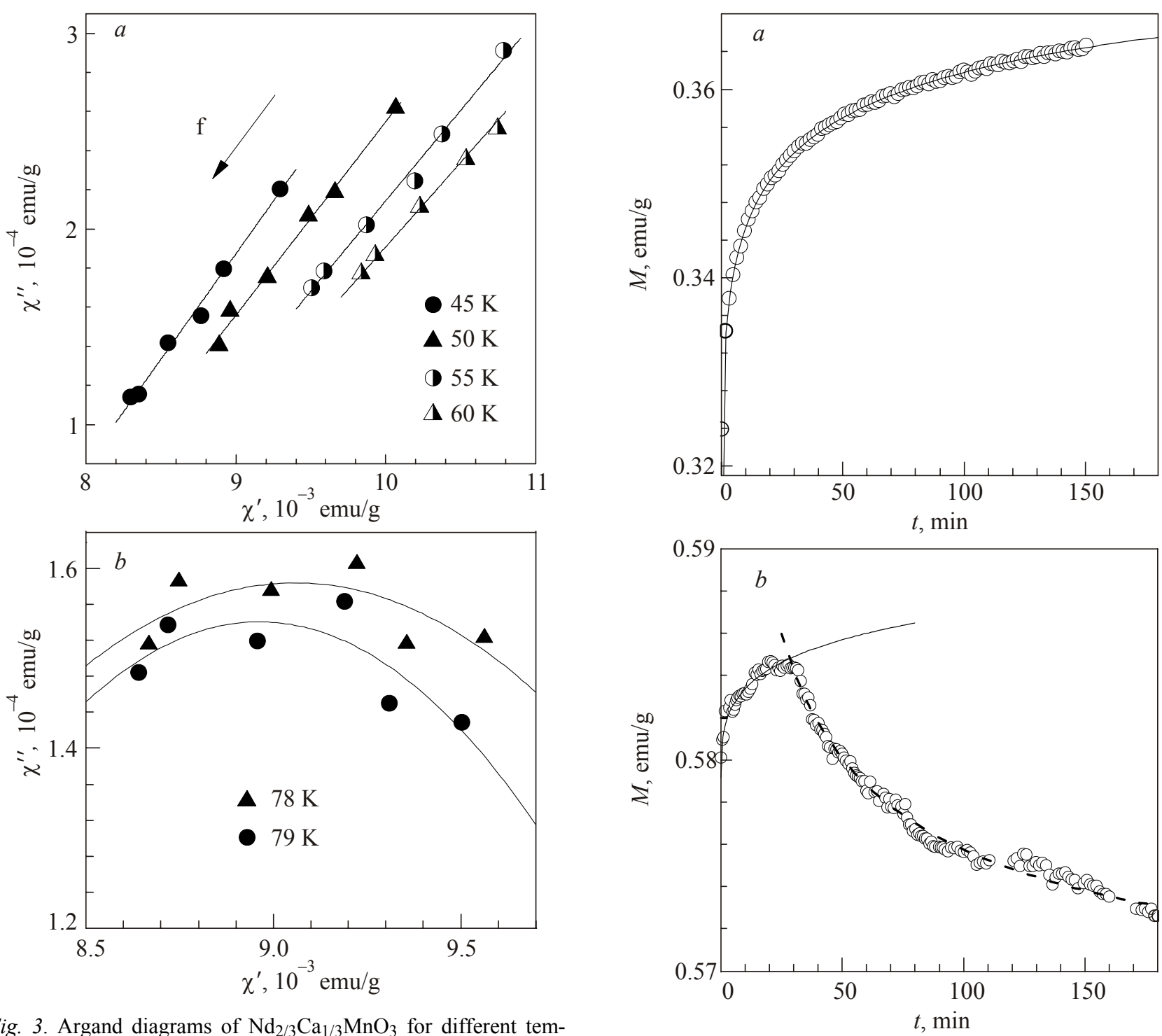

Fig. 3. Argand diagrams of $\mathrm{Nd}_{2 / 3} \mathrm{Ca}_{1 / 3} \mathrm{MnO}_{3}$ for different temperatures below $(a)$ and above $(b)$ the freezing temperature $T_{g} \sim 60 \mathrm{~K}$. The lines are fits by Eq. (1).

at 10 and $79 \mathrm{~K}$. At $10 \mathrm{~K}$ there is a continuous increase of the magnetization even after $2.5 \mathrm{~h}$, during which about $25 \%$ of the $\mathrm{FC}$ magnetization (in $\mu_{0} H=10 \mathrm{mT}$ ) was reached. We have fitted the low-temperature $M(t)$ dependence by the follow equation [17]:

$$
M(t)=M(0)\left[1+\beta \exp \left(-(t / \tau)^{\alpha}\right)\right],
$$

where $M(0)$ is the magnetization at $t=0$ and $\tau$ is the characteristic relaxation time dependent on the $T$. We have obtained following fitting parameters: $M(0)=0.38 \mathrm{emu} / \mathrm{g}$, $\beta=0.184, \tau=24.9 \mathrm{~min}, \alpha=0.287$. It needs to be noted that the obtained characteristic relaxation time $\tau=24.9 \mathrm{~min}$ agrees well with the value obtained for the field-cooled magnetization relaxation $\tau=29 \min$ [9].

The long-time relaxation behavior of the ZFC magnetization observed at low temperatures is associated with the tendency of ferromagnetic clusters to align in the ap-

Fig. 4. The time dependence of ZFC magnetization for $\mathrm{Nd}_{2 / 3} \mathrm{Ca}_{1 / 3} \mathrm{MnO}_{3}$ at $10(a)$ and $79(b) \mathrm{K}$. The magnetization data were collected immediately after applying the magnetic field $\mu_{0} H=10 \mathrm{mT}$. Solid line is the best fit of the magnetic relaxation by Eq. (2). Dash line is a guide for eyes only. The peak on the curve at $T=79 \mathrm{~K}$ is related to the competition between two different relaxation mechanisms.

plied magnetic field. Such relaxation agrees well with the very broad distribution of relaxation times resulting from the Cole-Cole analysis of dynamic magnetization, indicating that magnetic moments of coexisting clusters are frozen at macroscopic time scale.

At $79 \mathrm{~K}$, which is above $T_{g}$, the $M(t)$ curve shows a positive slope at the beginning $(\sim 20 \mathrm{~min})$ of the time run, indicating that the FM contribution associated with ferromagnetic clusters rises. Note that the intensity of the magnetization process and the value of the achieved magnetization are substantially lower than at $10 \mathrm{~K}$, which suggests a decreased value of the ferromagnetic fraction and a smaller size of ferromagnetic clusters at this temperature. These 
clusters can be associated with a residual ferromagnetic phase retained at time of measurement. The magnetization reaches its maximum after approximately $20 \mathrm{~min}$ and then starts to decrease. This curious peak on $M(t)$ observed at $79 \mathrm{~K}$ is related to the competition between two different relaxation mechanisms, the "up" relaxation one and the "down" relaxation one. The "up" relaxation process dominates at the beginning of the relaxation, while the "down" process dominates after $20 \mathrm{~min}$ at $79 \mathrm{~K}$. The "up" process was fitted by the Eq. (2), the same as was used for the relaxation of the magnetization at $10 \mathrm{~K}$. The best fitting parameters are $M(0)=0.57 \mathrm{emu} / \mathrm{g}, \beta=3.055, \tau=174 \mathrm{~min}$, $\alpha=0.114$. The nature of the "down" relaxation process is not quite clear, it may be related to the thermal excitations and needs to be further studied.

The long-time relaxation behavior found at low temperatures is consistent with a cluster magnetic glass state of the compound. It strongly reminds a magnetic behavior and an unusual long relaxation time of interacting magnetic nanoparticle systems $[18,19]$.

\section{Conclusions}

In the present study we have revealed a giant exchange bias in the colossal magnetoresistance $\mathrm{Nd}_{2 / 3} \mathrm{Ca}_{1 / 3} \mathrm{MnO}_{3}$ perovskite at low-temperatures, evident of the exchange coupling in the compound. The phenomena found confirm our previous assumption that the low-temperature magnetic structure of the compound are represented by small (nanosized) ferromagnetic clusters immersed in the chargeordered antiferromagnetic matrix. Magnetic behavior of the $\mathrm{Nd}_{2 / 3} \mathrm{Ca}_{1 / 3} \mathrm{MnO}_{3}$ perovskite is consistent with a cluster-glass magnetic state and does not agree with a classical spin-glass state observed in a variety of disordered magnetic systems. The cluster-glass magnetic behavior of $\mathrm{Nd}_{2 / 3} \mathrm{Ca}_{1 / 3} \mathrm{MnO}_{3}$ originates from a self-organized phaseseparated state of the compound.

The two strongly different relaxation mechanisms of the both ac and dc magnetizations have been found: the first one is characteristic for the temperatures below the freezing one $T_{g} \sim 60 \mathrm{~K}$, the second one is characteristic for temperatures above $T_{g}$.

The Cole-Cole analysis of the dynamic susceptibility at low temperature has shown extremely broad distribution of relaxation times, indicating that spins are frozen at "macroscopic" time scale. Slow relaxation in the zero-field-cooled magnetization has been experimentally revealed as well. The slow relaxation found confirms the cluster-glass magnetic state of the compound.

\section{Acknowledgments}

This paper is dedicated to Professor Viktor Valentinovich Eremenko on the occasion of his 80th birthday. One of authors (A.F.) is very thankful to Prof. Eremenko for decades of fruitful collaboration. Viktor Valentinovich was and still is an excellent mentor in the field of magnetism for the whole Košice Low Temperature Group.

Authors are thankful to Dr. D. Sheptyakov (PSI, Switzerland) and to Dr. D. Khalyavin (ISIS, United Kingdom) for fruitful collaboration.

This work was supported by the SAS Centre of Excellence CFNT MVEP, by the ERDF EU (European Union European regional development fund) grant under the contract No. TMS26220120005 and by the Slovak Grant Agency VEGA-1/0159/09. The financial support of U.S. Steel Košice is gratefully acknowledged.

1. A.B. Beznosov, E.L. Fertman, and V.A. Desnenko, Fiz. Nizk. Temp. 34, 624 (2008) [Low Temp. Phys. 34, 624 (2008)].

2. E. Fertman, D. Sheptyakov, A. Beznosov, V. Desnenko, and D. Khalyavin, J. Magn. Magn. Mater. 293, 787 (2005).

3. E. Fertman, A. Beznosov, D. Sheptyakov, V. Desnenko, M. Kajňaková, and A. Feher, J. Magn. Magn. Mater. 321, 316 (2009).

4. A. Beznosov, E. Fertman, V. Desnenko, M. Kajňaková, and A. Feher, J. Magn. Magn. Mater. 323, 2380 (2011).

5. E. Dagotto, Nanoscale Phase Sepatation and Colossal Magnetoresistance, Springer Series in Solid State Physics, Springer-Verlag, Berlin (2003).

6. F. Rivadulla, M.A. López-Quintela, and J. Rivas, Phys. Rev. Lett. 93, 167206 (2004).

7. D. Niebieskikwiat, J. Tao, J.M. Zuo, and M.B. Salamon, Phys. Rev. B78, 014434 (2008).

8. J. Dho, W.S. Kim, and N.H. Hur, Phys. Rev. Lett. 89, 027202 (2002).

9. A. Feher, M. Kajňaková, E.L. Fertman, A.B. Beznosov, V.A. Desnenko, and S.N. Dolya, Nanosystems, Nanomaterials, Nanotechnologies 9, 189 (2011).

10. D. Niebieskikwiat and M.B. Salamon, Phys. Rev. B72, 174422 (2005).

11. C. Dekker, A.F.M. Arts, and H.W. de Wijn, A.J. van Duyneveldt, and J.A. Mydosh, Phys. Rev. B40, 11243 (1989).

12. T. Mori and H. Mamiya, Phys. Rev. B68, 214422 (2003).

13. J.A. Mydosh, Spin-Glasses: An Experimental Introduction, Taylor and Francis, London (1993).

14. S. Chikuzami, Physics of Ferromagnetism, Clarendon, Oxford (1997).

15. J. Wu and C. Leighton, Phys. Rev. B67, 174408 (2003).

16. I.G. Deac, J.F. Mitchell, and P. Schiffer, Phys. Rev. B63, 172408 (2001).

17. Xi Chen, S. Sahoo, W. Kleemann, S. Cardoso, and P.P. Freitas, Phys. Rev. B70, 172411 (2004).

18. V. Markovich, I. Fita, A. Wisniewski, G. Jung, D. Mogilyansky, R. Puzniak, L. Titelman, and G. Gorodetsky, Phys. Rev. B81, 134440 (2010).

19. Y. Sun, M.B. Salamon, K. Garnier, and R.S. Averback, Phys. Rev. Lett. 91, 167206 (2003). 